\title{
Synthesis and characterization of MEH-PPV/nanosized titania hybrids prepared via in situ sol-gel reaction
}

\author{
Hung-Jen Chen ${ }^{a}$, Leeyih Wang ${ }^{\mathrm{b}, \mathrm{c}, *, 1}$, Wen-Yen Chiu ${ }^{\mathrm{a}, \mathrm{c}, *}$ \\ a Department of Chemical Engineering, National Taiwan University, Taipei, Taiwan, ROC \\ b Center for Condensed Matter Sciences, National Taiwan University, Taipei, Taiwan, ROC \\ ' Institute of Polymer Science and Engineering, National Taiwan University, Taipei, Taiwan, ROC
}

\section{A R T I C L E I N F O}

\section{Article history:}

Received 7 May 2007

Received in revised form 29 May 2008

Accepted 4 June 2008

\section{Keywords:}

MEH-PPV/titania hybrid

In situ sol-gel reaction

Morphology

\begin{abstract}
A B S T R A C T
Homogenous organic/inorganic hybrids (MEH-PPV/nanosized-titania) were successfully synthesized via in situ sol-gel reaction, using titanium isopropoxide (TIP) as precursor. These hybrids were prepared under various weight ratios of MEH-PPV/TIP/ $\mathrm{H}_{2} \mathrm{O} / 2$-chlorophenol and different molecular weights of MEH-PPV, whose effects on hybrids' properties were studied. The hybrids were characterized by X-ray diffraction (XRD), thermogravimetric analysis (TGA), and scanning electron microscopy (SEM). XRD showed clearer crystal patterns at higher conversion of TIP to titania. TGA showed higher residual weight at higher conversion of TIP to titania. At molar ratio of water to TIP of 4.0 and MEH-PPV concentration of $5 \mathrm{mg} / \mathrm{ml}$, homogenous films of the hybrids were observed from SEM, in which the amount of TIP could be as high as $89 \mathrm{wt} . \%$.
\end{abstract}

(C) 2008 Elsevier B.V. All rights reserved.

\section{Introduction}

The preparation of organic/nanosized-inorganic hybrid is attracting a great deal of interest due to its unique ability to combine the remarkable advantages of organic and inorganic materials which increase its potential for application. However, the essentially different chemical natures of organic and inorganic materials are the main challenge for the preparation of homogenous organic/inorganic hybrids with high performance. Many approaches have been used in attempts to improve the compatibility of organic and inorganic materials. Among the various approaches under development, the preparation method based on in situ reaction, either in situ polymerization or in situ sol-gel reaction, has been the widely adopted technique because it enables good control of the miscibility of organic and inorganic components at the molecular, micro, or macro-scale [1,2].

The general route of in situ sol-gel reaction was to functionalize organic polymer backbone with a silane coupling agent, trialkoxysilane moieties. These functionalized polymers proceeded hydrolysis and condensation reaction with metal alkoxide, and an interpenetrating network formed between organic and inorganic components. The most widely used metal alkoxide is tetraethoxysi-

\footnotetext{
* Corresponding author. Tel.: +886 23623259; fax: +8862 23623259.

E-mail addresses: leewang@ntu.edu.tw (L. Wang) ycchiu@ntu.edu.tw (W.-Y. Chiu).

1 Tel.: +8862 33665276; fax: +886223655404
}

lane (TEOS). For different purposes, different functional metal alkoxides could also be introduced into the organic polymer phase through a similar process. Nevertheless, the condition for incorporating metal alkoxides with a higher reaction rate, such as $\mathrm{Ti}(\mathrm{OR})_{4}$ or $\mathrm{Zr}(\mathrm{OR})_{4}$ [3], was more difficult to control. Qin et al. successfully prepared a transparent and yellowish poly(methyl acrylate-co-itaconic anhydride) $/ \mathrm{TiO}_{2}$ hybrid by using 3-aminopropyltriethoxysilane (ATPES) as a coupling agent [4]. The film thickness, refractive index, and optical transparency could be controlled by the amount of titania and the species of coupling agent used $[5,6]$.

Poly[2-methoxy-5-(2'-ethylhexyloxy)-p-phenylenevinylene] (MEH-PPV), first synthesized by Wudl and Srdanov [7], has been studied for application in PLED [8], FET [9], and solar cell [10]. The aim of this study is to provide a simple way to prepare homogenous MEH-PPV/titania hybrid films. These were prepared via an in situ sol-gel reaction of titanium isopropoxide (TIP) in MEH-PPV solution without addition of coupling agents, in which there were essential interactions existing between MEH-PPV and TIP $[11,12]$. The effects of preparation conditions, e.g. water and TIP contents, concentration and molecular weight of MEH-PPV, on the properties and microstructure of the hybrids were discussed.

\section{Experimental \\ 2.1. Materials}

Titanium (IV) isopropoxide (TIP) and 2-chlorophenol (2-CP) were used as received. The water used in the sol-gel reaction was purified using a Millipore Milli-Q system. The weight-average molecular weights (Mw) of the two MEH-PPV 
Table 1

Composition of MEH-PPV/titania hybrids in sol-gel reaction

\begin{tabular}{|c|c|c|c|c|}
\hline & Mw of MEH-PPPV & H2O/TIP (molar ratio) & Titania(TIP)/hybrid (wt.\%) & Concentration of MEH-PPV in 2-CP $(\mathrm{mg} / \mathrm{ml})$ \\
\hline hiM-W0-T85-C5 & 600,000 & 0 & $85(95.2)$ & 5 \\
\hline hiM-W2-T85-C5 & 600,000 & 2 & $85(95.2)$ & 5 \\
\hline hiM-W4-T85-C5 & 600,000 & 4 & $85(95.2)$ & 5 \\
\hline hiM-W16-T85-C5 & 600,000 & 16 & $85(95.2)$ & 5 \\
\hline hiM-W4-T85-C5 & 600,000 & 4 & $85(95.2)$ & 5 \\
\hline hiM-W4-T70-C5 & 600,000 & 4 & $70(89.2)$ & 5 \\
\hline hiM-W4-T50-C5 & 600,000 & 4 & $50(77.8)$ & 5 \\
\hline hiM-W4-T30-C5 & 600,000 & 4 & $30(60.3)$ & 5 \\
\hline hiM-W4-T85-C5 & 600,000 & 4 & $85(95.2)$ & 5 \\
\hline hiM-W4-T85-C3 & 600,000 & 4 & $85(95.2)$ & 3 \\
\hline hiM-W4-T85-C1 & 600,000 & 4 & $85(95.2)$ & 1 \\
\hline loM-W4-T85-C5 & 50,000 & 4 & $85(95.2)$ & 5 \\
\hline loM-W4-T70-C5 & 50,000 & 4 & $70(89.2)$ & 5 \\
\hline hiM-W4-T85-C5 & 600,000 & 4 & $85(95.2)$ & 5 \\
\hline hiM-W4-T70-C5 & 600,000 & 4 & $70(89.2)$ & 5 \\
\hline
\end{tabular}

polymers were $\sim 600,000$ and $\sim 50,000$, respectively, as determined by gel permeation chromatography (GPC) and calibrated using polystyrene standard. The MEH-PPV was stored in the dark when not in use.

\subsection{Preparation and characterization procedure of $M E H-P P V / t i t a n i a(T I P)$ hybrid}

Table 1 shows the reagents and their amount used in the preparation of MEHPPV/titania(TIP) hybrids via in situ sol-gel reaction. Taking "hiM-W2-T85-C5" as an example, the first term hiM represents the molecular weight $(\sim 600,000)$ of MEH-PPV. The second term refers to the water amount and 2 represents the
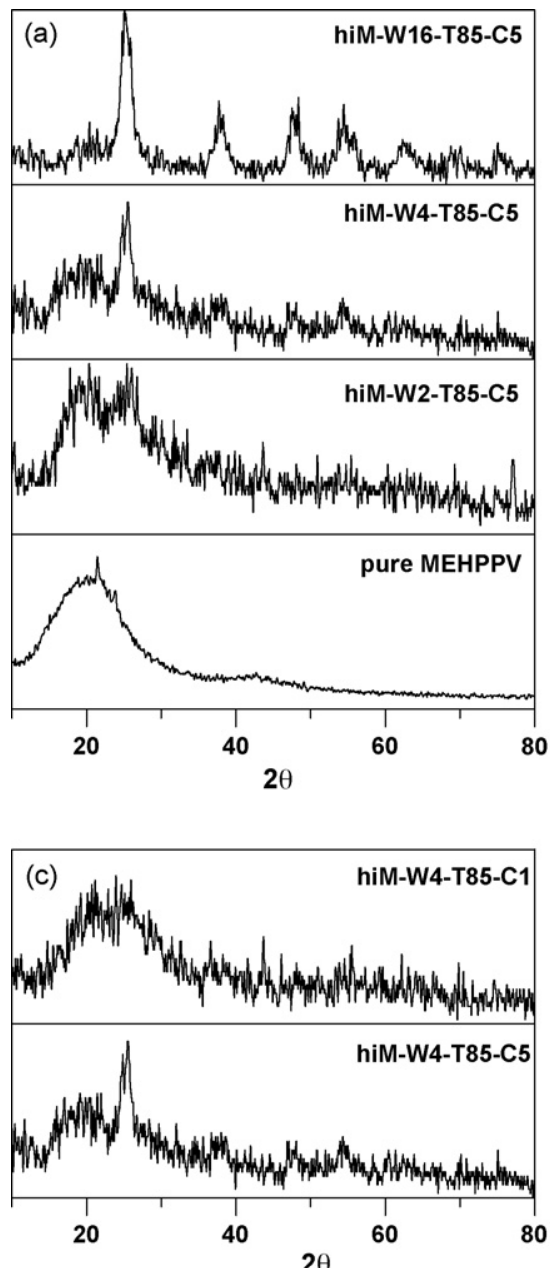

molar ratio of water to TIP. The third term refers to the TIP amount and 85 represents the weight percent of $\mathrm{TiO}_{2}$ in the hybrid if the conversion of TIP to $\mathrm{TiO}_{2}$ was $100 \%$. To obtain $85 \mathrm{wt} . \%$ of titania in the hybrid, the real wt.\% of TIP participating in the in situ sol-gel reaction was determined to be $95.2 \mathrm{wt} . \%$. The last term refers to the solution concentration, $5 \mathrm{mg} / \mathrm{ml}$, of MEH-PPV in 2 CP.

A $100 \mathrm{ml}$ round-bottom flask equipped with stirrer and condenser was used. In a typical procedure, the desired amount of TIP was fully dissolved in a premixed solution containing MEH-PPV and 2-CP. After 30 min stirring, the calculated amount of water was added drop wise in the MEH-PPV/TIP solution. The sol-gel
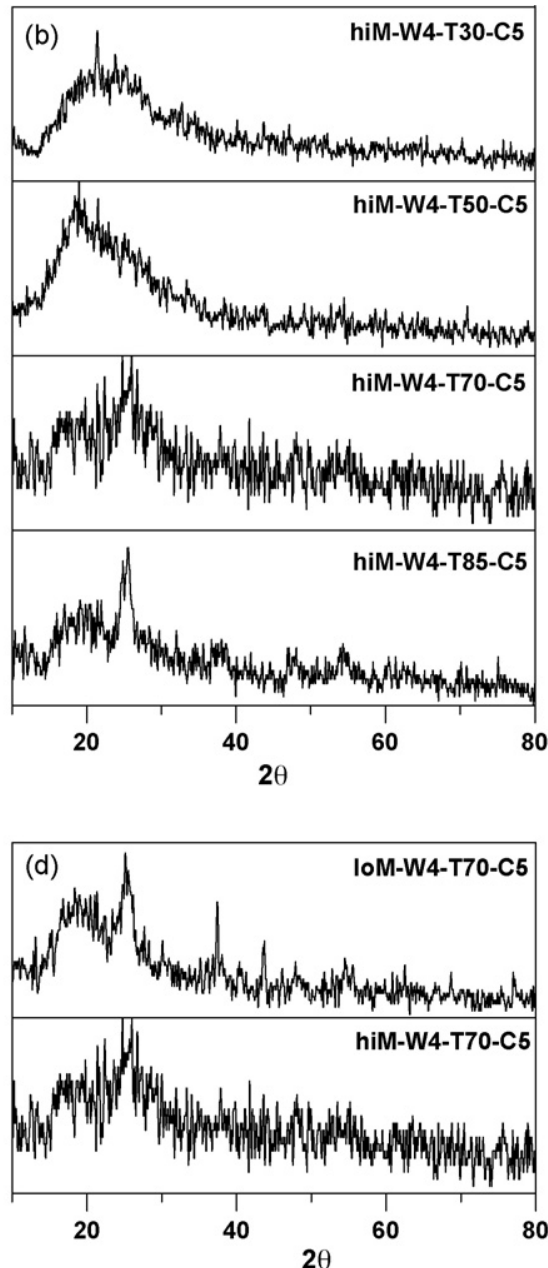

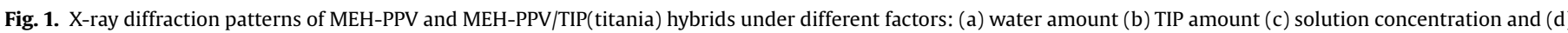
molecular weight of MEH-PPV. 
reaction was carried out first at room temperature for $30 \mathrm{~min}$ and then at $90^{\circ} \mathrm{C}$ for $10 \mathrm{~h}$.

Before the following analysis, the solvent in the prepared hybrids should be evacuated under $100^{\circ} \mathrm{C}$ vacuum oven over $48 \mathrm{~h}$. The measured samples were ground into a powder form for the analysis. An X-ray diffraction experiment (MAC Science Diffractometer MXP-3), using $\mathrm{Cu} K \alpha$ radiation at $40 \mathrm{kV}$ and $30 \mathrm{~mA}$, was conducted to examine the crystal pattern of MEH-PPV/titania(TIP) hybrids. A thermogravimetric analyzer (TGA) from PerkinElmer (TGA-7) was used to investigate the thermal degradation behavior of MEH-PPV/titania(TIP) hybrids. Specimens of 5-15 mg ground powder were put into a platinum crucible and heated at a rate of $10^{\circ} \mathrm{C} / \mathrm{min}$ under nitrogen atmosphere. The recorded temperature was from 100 to $800^{\circ} \mathrm{C}$. The aggregate size and aggregation phenomena of MEH-PPV/titania(TIP) hybrids were observed by Hitachi (S-800) Scanning Electronic Microscope (SEM) at an activation voltage of $20 \mathrm{kV}$.

\section{Results and discussion}

The interaction between MEH-PPV and TIP was a key point to prepare a homogenous MEH-PPV/titania hybrid. In titanium alkoxides, the oxidation state $n(=4)$ of the metal is lower than its normal coordination number $N(=6)$. Therefore, full coordination of Ti is not satisfied in the monomeric alkoxide $\mathrm{Ti}(\mathrm{OR})_{4}$. So, Ti prefers to increase its coordination number by using its empty d orbital to accept oxygen or nitrogen lone-pairs from nucleophilic ligands [13]. Besides, from our previous study, it was indicated that TIP interacted with MEH-PPV from the d orbital of Ti atom and the electron lone pair of $\mathrm{O}$ atom of MEH-PPV [11]. Thus, the sol-gel reaction of TIP was effectively slowed down to avoid phase separation from MEH-PPV.

\subsection{XRD analysis}

The crystal patterns of the hybrids with different compositions were shown in Fig. 1 through XRD measurement.

\subsubsection{Effect of water amount}

Fig. 1(a) showed that the crystallinity of titania increased as the amount of water in the sol-gel reaction was increased, due to the larger amount of water accelerating the sol-gel reaction of TIP and promoting the growth of titania.

\subsubsection{Effect of TIP amount}

In Fig. 1(b), the crystallinity of titania decreased as the amount of TIP was decreased. The crystallinity decrease was due to the sol-gel reaction being significantly slowed down by two effects: (1) low concentration of TIP and (2) higher interaction of TIP with MEH-PPV [11].

\subsubsection{Effect of solution concentration}

Fig. 1(c) showed that the crystallinity of titania increased as the sol-gel reaction took place in a more concentrated solution. MEH-PPV chains were in coiled conformation in more concentrated solution, so that the interaction of TIP and MEH-PPV decreased. Both effects, concentration and interaction, caused the conversion of TIP to titania to be higher, and thus a higher crystallinity of titania was observed.
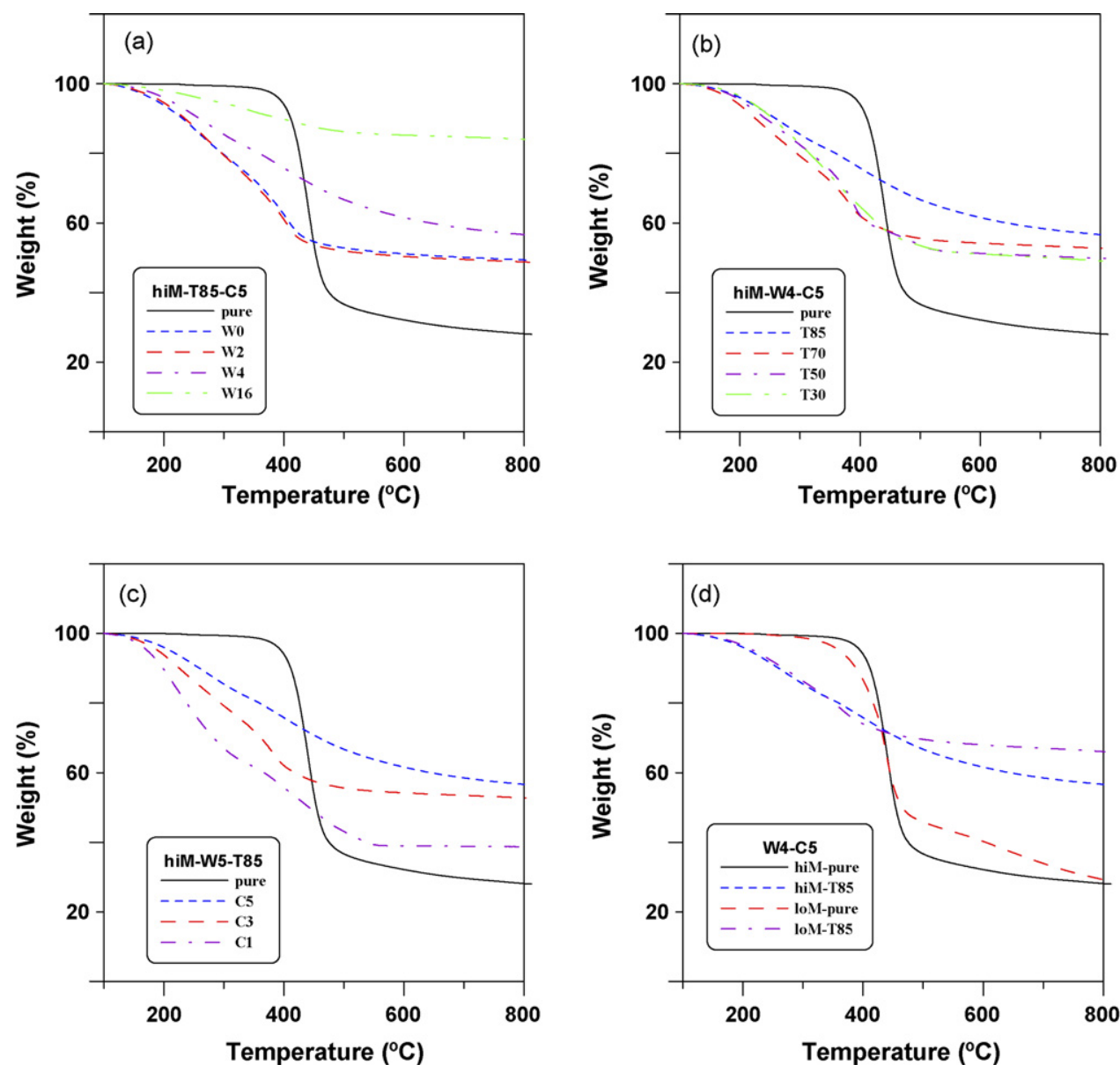

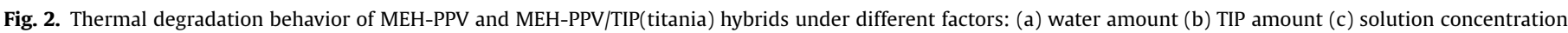
and (d) molecular weight of MEH-PPV. 


\subsubsection{Effect of molecular weight of MEH-PPV}

Fig. 1(d) showed the effect of molecular weight of MEH-PPV on the X-ray diffraction patterns of hybrids. The crystallinity of titania in the hybrid containing higher Mw MEH-PPV was lower than that in hybrid containing lower Mw MEH-PPV. Because the hybrid solution containing higher Mw MEH-PPV had higher viscosity, it limited the growth of titania.

\subsection{Thermal analysis}

Fig. 2(a)-(d) showed the thermal degradation behaviors of hybrids and pure MEH-PPV, respectively. All hybrids exhibited lower thermal stability initially in a TGA curve than pure MEH-PPV, which was mainly attributed to the weight loss of transformation of the un-reacted groups, such as $\mathrm{Ti}-\mathrm{OR}$ and $\mathrm{Ti}-\mathrm{OH}$, into $\mathrm{Ti}-\mathrm{O}-\mathrm{Ti}$ bonds. As the temperature is increased, the hybrid exhibited higher thermal stability than pure MEH-PPV, which was ascribed to the newly formed inorganic network.

\subsubsection{Effect of water amount}

Fig. 2(a) showed the effect of water amount on the thermal degradation behavior of hybrids. The thermal stability increased as the amount of water was increased. The larger amount of water promoted the growth of titania; therefore, it raised the thermal stability of the hybrid.

\subsubsection{Effect of TIP amount}

Fig. 2(b) showed the effect of TIP amount on the thermal degradation behavior of the hybrid. The thermal stability of hiMW4-T85-C5 was higher and the other three hybrids exhibited similar thermal degradation behavior. The conversion of TIP to titania was higher for hiM-W4-T85-C5 as discussed above. Therefore, the thermal stability of hiM-W4-T85-C5 was higher than the others.

\subsubsection{Effect of solution concentration}

Fig. 2(c) showed the thermal degradation behavior of hybrids prepared from different solution concentrations. According to the XRD analysis, the conversion of TIP to titania decreased as the solution concentration decreased. Therefore, hiM-W4-T85-C1 retained a higher amount of $\mathrm{Ti}-\mathrm{OR}$ and $\mathrm{Ti}-\mathrm{OH}$ un-reacted groups; the thermal stability of hiM-W4-T85-C1 was the lowest.

\subsubsection{Effect of molecular weight of MEH-PPV}

Fig. 2(d) showed the effect of molecular weight of MEHPPV on the thermal degradation behavior of hybrids. The hybrid with lower Mw MEH-PPV had a higher residual weight at $800^{\circ} \mathrm{C}$ than the hybrid with higher Mw MEH-PPV. According to the XRD analysis, the conversion of TIP to titania was higher in the hybrid with lower Mw MEH-PPV. It resulted in a higher thermal stability.

\subsection{SEM morphology}

The particle size of TIP(titania) in the MEH-PPV/TIP(titania) hybrid could be observed from the SEM photograph. The resulted particles size and distribution were discussed according to the effect of water amount, TIP amount, solution concentration, and molecular weight of MEH-PPV. The symbol $\left(^{*}\right)$ labeled in the figure meant that it was 1000 times magnified. If no symbol $\left({ }^{*}\right)$ was labeled, the figure was with 50,000 magnification.

\subsubsection{Effect of water amount}

Fig. 3(a)-(d) showed that titania particles were growing and aggregating in size, and phase separation gradually occurred as the water amount was increased. As shown in XRD and TGA
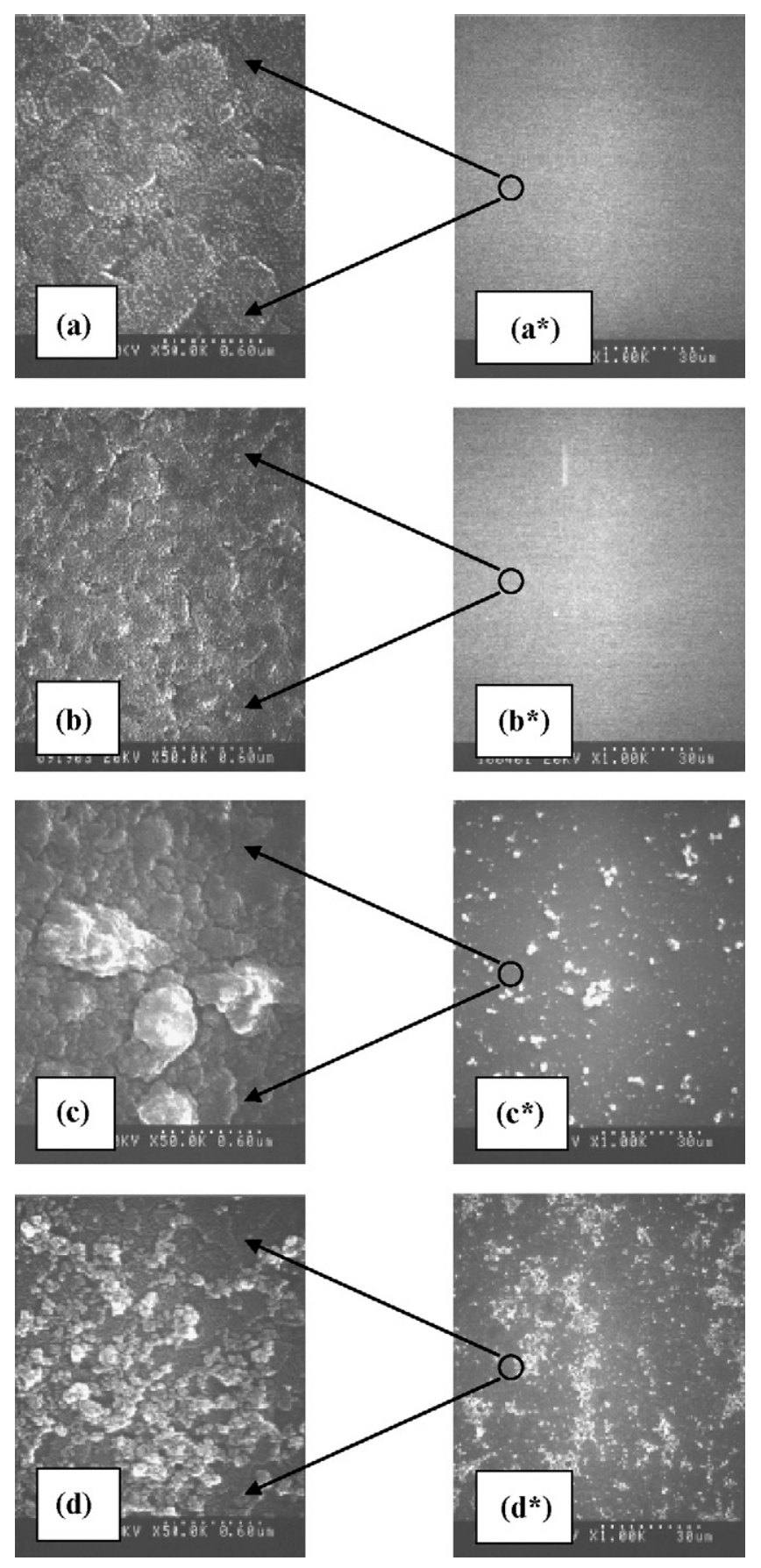

Fig. 3. SEM photographs of hybrid hiM-T85-C5 prepared with different amount of water: molar ratio of $\mathrm{H}_{2} \mathrm{O} / \mathrm{TIP}(\mathrm{a}), 0$ (b), 2 (c), 4 and (d) 16. The symbol ( $\left.{ }^{*}\right)$ meant 1000 times of magnification. Without $\left({ }^{*}\right)$, the figures were with 50,000 magnification.

analysis, the conversion of TIP to titania increased with the increase of water amount. In Fig. $3(a)^{*}$ and $(b)^{*}$, homogenous morphology was observed for hiM-W0-T85-C5 and hiM-W2-T85$\mathrm{C} 5$, in which the average size of titania particles was smaller than $10 \mathrm{~nm}$. In Fig. $3(\mathrm{c})^{*}$ and $(\mathrm{d})^{*}$, as the water amount was increased, phase separation between titania and MEH-PPV could be observed.

\subsubsection{Effect of TIP amount}

Fig. 4(a)-(c) and Fig. 3(c) showed the effect of TIP amount on the degree of aggregation and distribution of titania particles in 

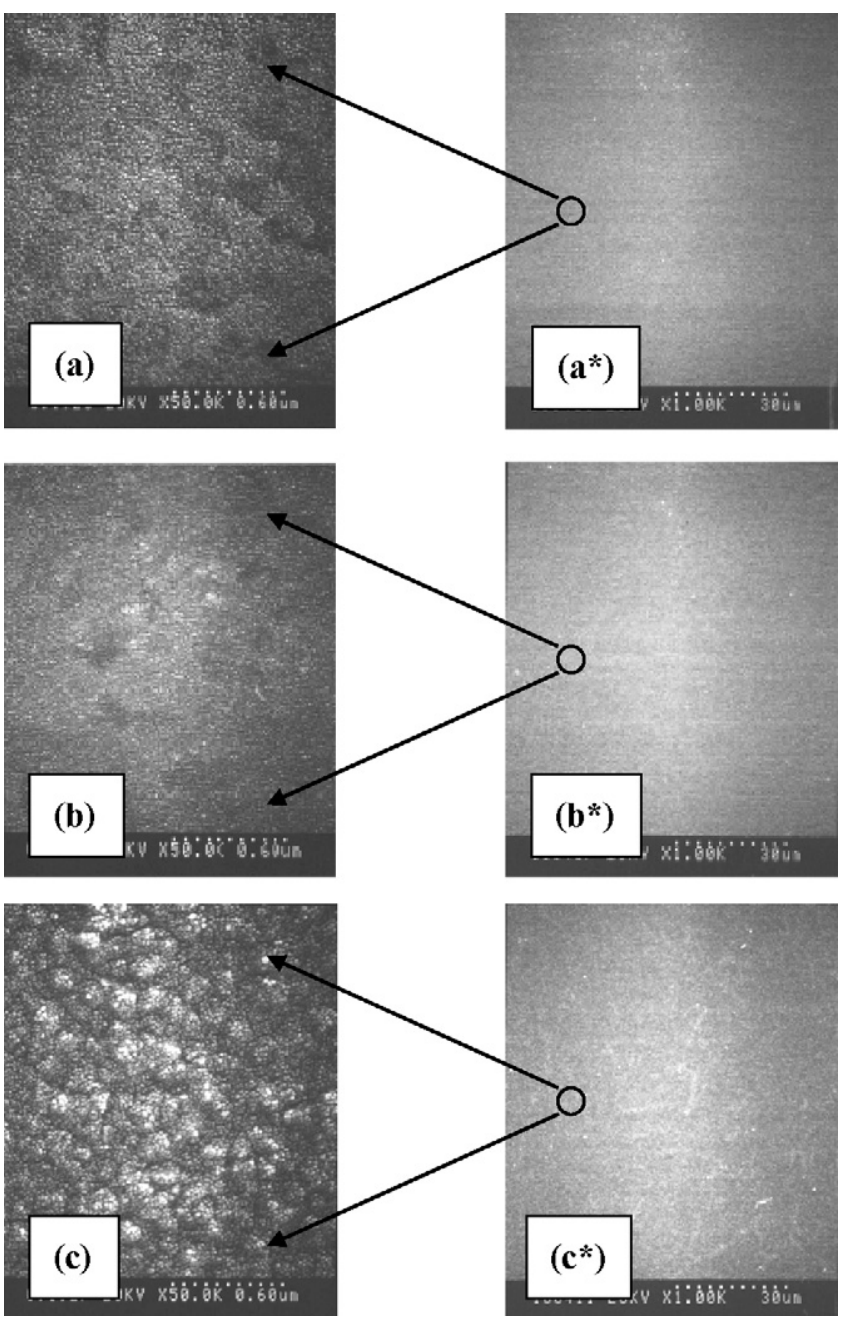

Fig. 4. SEM photographs of hybrids hiM-W4-C5 prepared with different weight percentages of TIP: (a) $60.3 \mathrm{wt} . \%$, (b) $77.8 \mathrm{wt} . \%$ and (c) $89.2 \mathrm{wt} . \%$. The symbol (*) meant 1000 times of magnification. Without $\left({ }^{*}\right)$, the figures were with 50,000 magnification.

the hybrids. The degree of aggregation increased as the amount of TIP was increased. In Fig. $4(\mathrm{a})^{*}-(\mathrm{c})^{*}$, homogenous morphology was observed. The size of titania particles was as small as $10-100 \mathrm{~nm}$. For the hybrid hiM-W4-T85-C5, the degree of aggregation of titania particles increased significantly, and phase separation was clearly seen in Fig. 3(c) ${ }^{*}$.

\subsubsection{Effect of solution concentration}

Figs. 5(a) and (b) and 3(c) were compared to investigate the degree of aggregation and distribution of titania particles in hybrids, which were prepared from different solution concentrations. The degree of aggregation decreased as the concentration of solution was decreased. In Fig. 5(a) and (b) ${ }^{*}$, homogenous morphology was observed. The size of titania particles was in the range of 10-250 nm for hiM-W4-T85-C1 and hiM-W4-T85-C3. However, in Fig. 3(c), the concentration of solution was higher and phase separation was obvious.

\subsubsection{Effect of molecular weight of MEH-PPV}

The SEM photographs of loM-W4-T70-C5 and hiM-W4-T70-C5 were shown in Fig. 6 for comparison. The effect of molecular weight of MEH-PPV on the morphology of the hybrids could be observed. The degree of aggregation of titania particles in the hybrid with
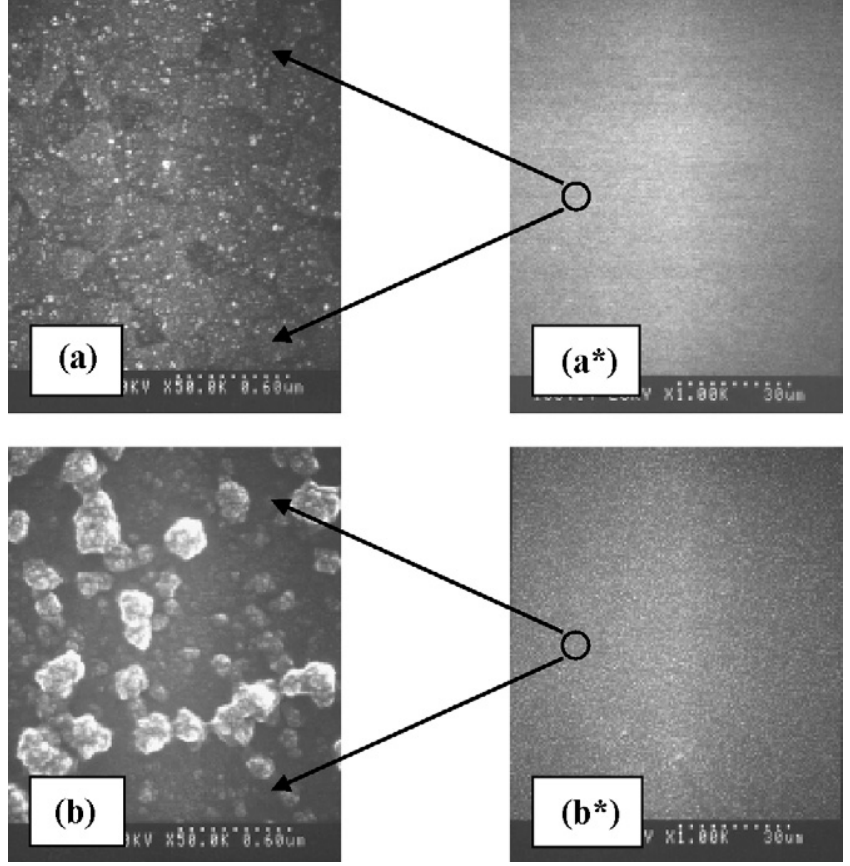

Fig. 5. SEM photographs of hybrids hiM-W4-T85 prepared with different concentrations of MEH-PPV: (a) $1 \mathrm{mg} / \mathrm{ml}$ and (b) $3 \mathrm{mg} / \mathrm{ml}$. The symbol $\left({ }^{*}\right)$ meant 1000 times of magnification. Without $\left({ }^{*}\right)$, the figures were with 50,000 magnification.

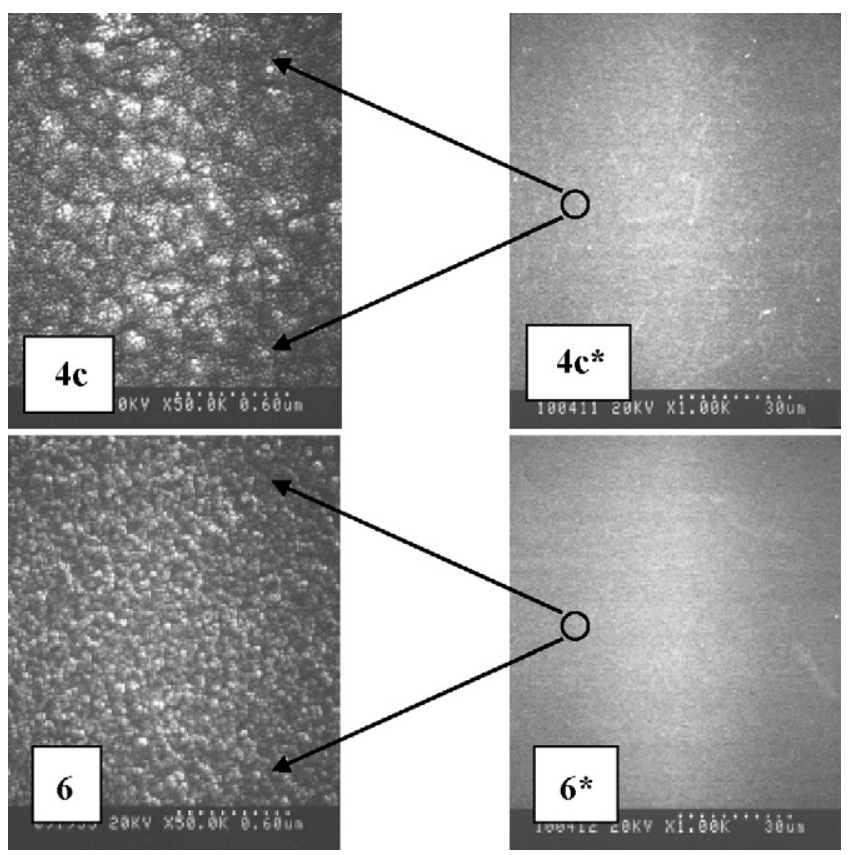

Fig. 6. SEM photographs of hybrids loM-W4-T70-C5 (6 and $\left.6^{*}\right)$ and hiM-W4-T70-C5 $\left(4 \mathrm{c}\right.$ and $4 \mathrm{c}^{*}$ ) showing the effect of Mw of MEH-PPV on the degree of aggregation and distribution of titania particles in hybrids. The symbol $\left({ }^{*}\right)$ meant 1000 times of magnification. Without $\left({ }^{*}\right)$, the figures were with 50,000 magnification.

higher Mw MEH-PPV was slightly higher, which was mainly due to the viscosity effect.

\section{Conclusions}

The crystal pattern, thermal degradation behavior, and morphology of MEH-PPV/TIP(titania) hybrids prepared via in situ sol-gel reaction were investigated based on different amounts 
of water, amounts of TIP, solution concentration, and molecular weight of MEH-PPV.

From XRD analysis, a higher amount of water accelerated the growth of titania. Hybrids with a higher amount of TIP exhibited a higher crystallinity of titania. The interaction between $\mathrm{MEH}-$ PPV and TIP increased in dilute solution, which slowed down the sol-gel reaction significantly and resulted in lower crystallinity of titania. The crystallinity of titania was higher in the hybrid with lower Mw MEH-PPV, compared to the hybrid with higher Mw MEH-PPV. Viscosity influenced the sol-gel reaction. From TGA analysis, the extent of transformation of TIP into $\mathrm{TiO}_{2}$ was responsible for the thermal stability of the hybrids. The degree of aggregation of titania particles in hybrids, observed from SEM photographs, increased with higher amounts of water, higher amounts of TIP, higher solution concentration, and higher molecular weight of MEH-PPV.

Homogenous morphology of hybrids could be obtained when the amount of water was not in great excess, the amount of TIP was lower than $89 \mathrm{wt} . \%$, the MEH-PPV concentration was lower than $3 \mathrm{mg} / \mathrm{ml}$, and the molecular weight of MEH-PPV was not too high.

\section{Acknowledgements}

The authors would like to thank Ching-Yen Lin, Chih-Yuan Tang, and the Instrumentation Center at National Taiwan University for the use of SEM.

\section{References}

[1] M.I. Sarwar, Z. Ahmad, Eur. Polym. J. 36 (2000) 94.

[2] R.A. Zoppi, C.R. de Castro, I.V.P. Yoshida, S.P. Nunes, Polymer 38 (1997) 5705

[3] B.M. Novak, Adv. Mater. 5 (1993) 422.

[4] H.H. Qin, J.H. Dong, K.Y. Qiu, Y. Wei, J. Appl. Polym. Sci. 78 (2000) 1763.

[5] E.J. Nassar, R.R. Goncalves, M. Ferrari, Y. Messaddeq, S.J.L. Ribeiro, J. Alloys Compd. 344 (2002) 221.

[6] W. Que, Y. Zhou, Y.L. Lam, Y.C. Chan, C.H. Kam, Thin Solid Films 358 (2000) 16

[7] F. Wudl, G. Srdanov, U.S. Patent 5,189,136, 1993.

[8] T.F. Guo, F.S. Yang, Z.J. Tsai, T.C. Wen, S.N. Hsieh, Y.S. Fu, Appl. Phys. Lett. 87 (2005) 13504

[9] T. Sakanoue, E. Fujiwara, R. Yamada, H. Tada, Appl. Phys. Lett. 84 (2004) 3037.

[10] H. Wang, C.C. Oey, A.B. Djurišic, M.H. Xie, Y.H. Leung, K.K.Y. Man, W.K. Chan, A Pandey, J.M. Nunzi, P.C. Chui, Appl. Phys. Lett. 87 (2005) 23507.

[11] H.J. Chen, L. Wang, W.Y. Chiu, Polym. J. Sci. Part A: Polym. Chem. 46 (2008) 515

[12] H.J. Chen, L. Wang, W.Y. Chiu, Eur. Polym. J. 43 (2007) 4750.

[13] M. Camail, M. Humbert, A. Margaillan, A. Riondel, J.L. Vernet, Polymer 39 (1998) 6525. 